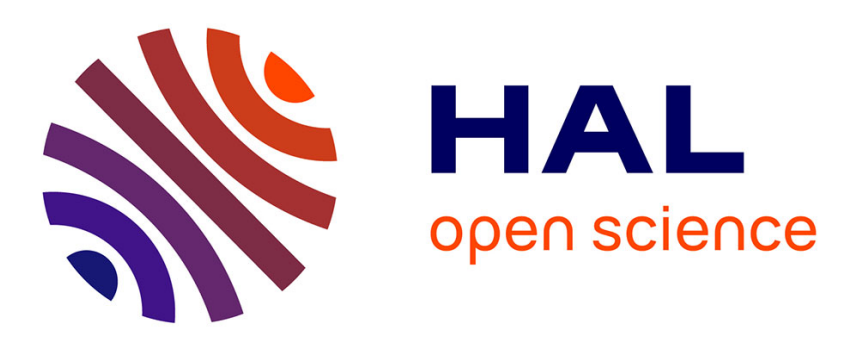

\title{
A heuristic procedure for solving the Lexicographic Bottleneck Assembly Line Balancing Problem (LB-ALBP)
}

Rafael Pastor, Ignacio Chueca, Alberto García-Villoria

\section{- To cite this version:}

Rafael Pastor, Ignacio Chueca, Alberto García-Villoria. A heuristic procedure for solving the Lexicographic Bottleneck Assembly Line Balancing Problem (LB-ALBP). International Journal of Production Research, 2011, pp.1. 10.1080/00207543.2011.578164 . hal-00720577

\section{HAL Id: hal-00720577 \\ https://hal.science/hal-00720577}

Submitted on 25 Jul 2012

HAL is a multi-disciplinary open access archive for the deposit and dissemination of scientific research documents, whether they are published or not. The documents may come from teaching and research institutions in France or abroad, or from public or private research centers.
L'archive ouverte pluridisciplinaire HAL, est destinée au dépôt et à la diffusion de documents scientifiques de niveau recherche, publiés ou non, émanant des établissements d'enseignement et de recherche français ou étrangers, des laboratoires publics ou privés. 


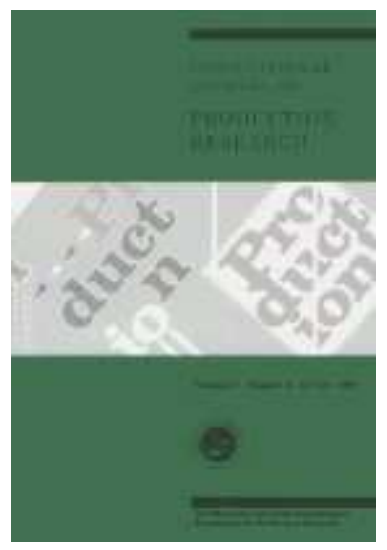

\section{A heuristic procedure for solving the Lexicographic Bottleneck Assembly Line Balancing Problem (LB-ALBP)}

\begin{tabular}{|r|l|}
\hline Journal: & International Journal of Production Research \\
\hline Manuscript ID: & TPRS-2010-IJPR-0962.R1 \\
\hline Manuscript Type: & Original Manuscript \\
\hline $\begin{array}{r}\text { Date Submitted by the } \\
\text { Author: }\end{array}$ & 26 -Feb-2011 \\
\hline Complete List of Authors: & $\begin{array}{l}\text { Pastor, Rafael; Universidad Politécnica de Cataluña, Instituto de } \\
\text { Organización y Control de Sistemas Industriales } \\
\text { Chueca, Ignacio; Universidad Politécnica de Cataluña } \\
\text { García-Villoria, Alberto; Universidad Politécnica de Cataluña, } \\
\text { Instituto de Organización y Control de Sistemas Industriales }\end{array}$ \\
\hline Keywords: & ASSEMBLY LINE BALANCING, ASSEMBLY LINES \\
\hline Keywords (user): & \multicolumn{2}{|c}{} \\
\hline \multicolumn{2}{|c}{}
\end{tabular}

\section{SCHOLARONE" \\ Manuscripts}




\title{
A heuristic procedure for solving the Lexicographic Bottleneck Assembly Line Balancing Problem (LB-ALBP) ${ }^{\dagger}$
}

\author{
Rafael Pastor*, Ignacio Chueca, Alberto García-Villoria \\ Institute of Industrial and Control Engineering (IOC) and Department of Management \\ Universitat Politècnica de Catalunya (UPC) \\ Av. Diagonal $647,11^{\text {th }}$ floor, 08028, Barcelona, Spain \\ rafael.pastor@upc.edu, igchueca@hotmail.com, alberto.garcia-villoria@upc.edu
}

\begin{abstract}
The Lexicographic Bottleneck Assembly Line Balancing Problem (LB-ALBP) is a new assembly line balancing problem which was recently defined in the literature. The LB-ALBP hierarchically minimizes the workload of the most heavily loaded workstation, followed by the workload of the second most heavily loaded workstation, followed by the workload of the third most heavily loaded workstation, and so on. The original study presents two mixed-integer linear programming (MILP) models designed to solve the LB-ALBP optimally, together with three heuristic procedures based on these MILPs. In this paper, we propose and test new algorithms that combine a heuristic procedure for obtaining an initial solution and several local search procedures, which are an improvement upon the heuristic procedures published to date.
\end{abstract}

Keywords: assembly line balancing

\section{Introduction}

Assembly lines are components of many production systems, such as those used in the automotive and household appliance industries. The classic assembly line balancing problem (ALBP) basically consists of assigning a set of tasks (each of them characterized by its processing time) to an ordered sequence of workstations, in such a way that the precedence constraints between the tasks are maintained and a given efficiency measure is optimized. Regarding the conventional terminology (e.g., Baybars, 1986), when the objective is to minimize the number of workstations for a given upper bound on the cycle time, the problem is known as ALBP-1; if the objective is to minimize the cycle time given a number of workstations, then the problem is called ALBP-2.

The problem of designing and balancing assembly lines has been extensively examined in the literature. A number of synthesis studies has been published, including the recent works of Rekiek et al. (2002), Becker and Scholl (2006), Scholl and Becker (2006) and Boysen et al. (2007, 2008); however, most papers focus on the simple case known as Simple ALBP (SALBP). This problem has been approached using heuristic and metaheuristic procedures e.g., Talbot et al. (1986), Ponnambalam et al. (1999) and Corominas and Pastor (2009);-, exact procedures based on binary or integer linear programming -e.g., White (1961), Talbot and Patterson (1984) and Pastor and Ferrer (2009)-, dynamic programming -e.g., Kao and Queyranne (1982)-, and branch and bound -e.g., FABLE by Johnson (1988), EUREKA by

\footnotetext{
† Supported by the Spanish Ministry of Education and Science, project DPI2007-61905, co-financed by FEDER.

* Corresponding author: Rafael Pastor, IOC Research Institute, Av. Diagonal, 647 (edif. ETSEIB), p. 11, 08028 Barcelona, Spain; Tel. + 34 9340117 01; fax + 349340166 05; e-mail: rafael.pastor@ upc.edu.
} 
Hoffman (1992) and SALOME by Scholl and Klein (1997)-. A significant variety of complex cases has been examined, including problems that consider lines with parallel workstations or parallel tasks; mixed or multi-models; multiple products; U-shaped, two-sided, buffered or parallel lines; incompatibility between tasks; stochastic processing times; and equipment selection -e.g., Park et al. (1997), Ağpak and Gökçen (2005), Amen (2006), Ding et al. (2006), Gamberini et al. (2006), Gökçen et al. (2006), Andrés et al. (2008), Corominas et al. (2008), Capacho et al. (2009), Corominas et al. (2010), Cortés et al. (2010) and Martino and Pastor (2010)-. As a result, generalized problems are becoming a widespread subject.

The ALBP-2 is a problem with a min-max objective, since it tries to minimize the workload of the most heavily loaded workstation (cycle time, $C T$ ). The scientific literature includes several problems with min-max objectives: location (Berman et al., 1994), production according to an ideal value (Yu, 1998), etc. The optimal solution of problems with this type of objective guarantees that the maximum value (the bottleneck) is as small as possible. However, the remaining values are not considered, and they can also be important because, even though they do not exceed the maximum value, their distribution can be highly irregular and therefore inappropriate. Indeed, in the ALBP, it is important to consider the secondbiggest, third-biggest, etc. workloads: the reliability of the line is improved; the workload is prone to be uniformly distributed among all workstations; and, moreover, as it is exposed in Boysen et al. (2006), the quality defects caused by stations with disproportionately large workstation times are avoided. Figure 1 shows that distributing a workload among six workstations as $10,10,10,4,3,3$ is not the same as distributing it as $10,6,6,6,6,6$; the cycle time value is the same, but the second distribution is beyond question more reliable than the first one and the workload is more uniformly distributed among the workstations.

\section{Insert Figure 1}

Pastor (2011) presents and formalizes the Lexicographic Bottleneck Assembly Line Balancing Problem (LB-ALBP), a new assembly line balancing problem. The LB-ALBP hierarchically minimizes the workload of the most heavily loaded workstation, followed by the workload of the second most heavily loaded workstation, followed by the workload of the third most heavily loaded workstation, and so on. It is therefore a multi-objective optimization problem with a hierarchical structure (e.g. Krajewski and Ritzman (1979) and Cortés et al. (2006)) in which the order of the objectives is the main point and there is no interest in a continuous trade-off among the functions. In general, the term "lexicographic optimization" is applied when different objectives must be optimized, but there is also literature that applies this term when the same objective is being optimized (e.g. Pentico, 2007). For more details about the lexicographic bottleneck objective, see, for example, Burkard and Rendl (1991) and Sokkalingam and Aneja (1998).

In Pastor (2011) two mixed-integer linear programming (MILP) models are proposed to optimally solve the LB-ALBP. The effectiveness of these two MILP models was evaluated by solving the benchmark instances that are usually used by the international scientific community to evaluate its algorithms (302 well-known SALBP-2 instances available on Scholl and Klein's homepage for assembly line balancing research, www.assembly-linebalancing.de). The two MILP models were run with a computing time limit of 18,000 seconds. The obtained results show that the aforementioned two MILP models found a 
solution which optimality is proven few times: specifically, 32 and 57 times, respectively, for the 302 benchmark instances.

Moreover, taking advantage of the designed mathematical programs, three heuristic procedures based on these MILPs were also designed and evaluated in Pastor (2011). The main idea consists of running the MILP models for 18,000 seconds and preserving the best solution obtained. The difference between the heuristics is the MILP model on which they are based and how the computing time is distributed when the mathematical submodels are sequentially solved. The effectiveness of the heuristic procedures was evaluated under the same conditions used with the MILP models, and the best results were obtained by the heuristic procedure called by the author H1_SHM.

As it is expounded in Pastor (2011), several papers have already dealt with multi-objective ALBPs, but the approach proposed in that paper is different. On the other hand, other objective functions also tend to homogeneously distribute the workload among all workstations, such as the "smoothness index" by Moodie and Young (1965), but the "lexicographic bottleneck" objective is different -as it has been introduced, for assignment problems, by Pentico (2007), and it is proved for the "smoothness index" objective in Pastor (2011).

In order to quantitatively compare two solutions of a LB-ALBP instance, Pastor (2011) designs a parameter, $\delta$, as a measurement of solution quality. $\delta$ considers, between both solutions, the difference in workload of each pair of workstations according to the list in descending order of workload:

$$
\delta=\frac{\Delta_{1} \cdot \beta^{m}+\Delta_{2} \cdot \beta^{m-1}+\ldots+\Delta_{m} \cdot \beta}{C T_{b e s t} \cdot \beta^{m-1}}
$$

where $\Delta_{j}$ is the positive, null or negative workload difference in the $j$-th most heavily loaded station between the worst and best solutions which are compared; $m$ is the number of workstations; $C T_{\text {best }}$ is the best cycle time of the two solutions compared; and $\beta$ is a parameter whose value must guarantee the hierarchy of the objectives. Pastor (2011) checked that the hierarchy of the objectives was guaranteed setting $\beta$ to 100 . For example, let us consider an instance with four workstations and the solutions $\mathrm{Sol}_{1}$ and $\mathrm{Sol}_{2}$, which have workloads, in descending order, of 50-48-46-44 and 50-47-46-45 time units, respectively. $\mathrm{Sol}_{2}$ is better than $\mathrm{Sol}_{1}$ because, although the cycle times are the same, $\mathrm{Sol}_{2}$ has a smaller workload in the second most heavily loaded station. The value $\delta$ associated with $S_{0} l_{1}$, with values of $\Delta_{j}$ equal to 0 (50-50), 1 (48-47), 0 (46-46) and -1 (44-45), is obtained as follows: $\delta=\frac{0 \cdot 100^{4}+1 \cdot 100^{3}+0 \cdot 100^{2}+(-1) \cdot 100}{50 \cdot 100^{3}}=0.02$. Thus, the worse a solution with respect to the reference solution, the larger the value of $\delta$.

In this paper, we propose and test new algorithms that combine a heuristic procedure for obtaining an initial solution and several local optimization procedures, which are an improvement upon heuristic procedures published to date. The remainder of the paper is 
organized as follows: Section 2 presents our heuristic procedure for solving the LB-ALBP; Section 3 describes the results of the subsequent computational experiment; and, finally, Section 4 is devoted to conclusions and possible lines for future research.

\section{A heuristic procedure for solving the LB-ALBP}

As it has been introduced, the original study of the LB-ALBP (Pastor, 2011) formalizes the problem and presents two mixed-integer linear programming (MILP) models designed to solve it optimally, together with three heuristic procedures based on these MILPs. In this paper, we propose and test new algorithms that combine a heuristic procedure for obtaining an initial solution and several local search procedures, which are an improvement upon the heuristic procedures published to date.

In Section 2.1 we first give a general overview of the idea in which is based our proposed heuristic followed by its formal description given in Section 2.2. The heuristic uses a local search at each iteration and the solution obtained may depend on the used local search; several local search procedures are proposed in Section 2.3.

\subsection{Introduction to the heuristic}

The heuristic is based on the heuristic resolution of several simple assembly line balancing problems of type 2 (SALBP-2). The main idea is to sequentially solve and divide the problem in smaller subproblems (i.e., subproblems with fewer stations and fewer tasks to assign) using the most loaded workstation as split point; then, each subproblem is solved as a SALBP-2. The obtained solution of each subproblem is used to update the global solution of the original problem. A core feature to the proposed heuristic is the application of local search procedures based on the trade and transfer of tasks between two workstations at each iteration of the heuristic.

To split iteratively the problem into smaller subproblems and to solve each of them as a SALBP-2 make possible to apply the procedures proposed in the literature for solving the SALBP-2 and, therefore, we can take advantage of the scientific research developed during years.

The following example is used to introduce the general idea of the heuristic. Let have 14 stations and $n$ tasks, and let the sum of the processing times $t_{i}(i=1, \ldots, n)$ of all tasks, $T$ $\left(T=\sum_{i=1 . . n} t_{i}\right)$, be equal to 324 . The heuristic first solves the original problem as a SALBP-2 and applies a local search into the obtained solution. Let assume that the solution obtained after applying the local search is the one shown in Figure 2a (let it be called $S T$ ). Next the most workloaded station is detected ( $4^{\text {th }}$ station) and the following two subproblems are generated: one that consists of the tasks that are assigned to the three first stations and considering three workstations (Subproblem 1), and a second subproblem that consists of the tasks that are assigned to the stations between the fifth and last ones and considering ten stations (Subproblem 2). 
At the next iteration, a subproblem is chosen; let suppose that the second generated subproblem is the chosen one (Subproblem 2). Then we solve it as a SALBP-2 and let suppose that the solution obtained for this subproblem is the one shown in Figure $2 b$ (let it be called $S T^{\prime}$ ). Next the global solution $S T$ is updated using $S T^{\prime}$ (Figure 2c) and two new subproblems are generated: one considering the tasks that are assigned to the stations between the fifth and the ninth ones of the updated $S T$ and with five stations (Subproblem 3), and another subproblem considering the tasks that are assigned to the stations between the eleventh and the fourteenth ones of the updated $S T$ and with four stations (Subproblem 4). Moreover, a local search is applied to the updated $S T$ and if a better solution than the best known is found (let it be called $S T^{*}$ ) then the procedure is reset starting from $S T^{*}$. This process of splitting iteratively each subproblem into smaller ones and solving them as a SALBP-2 is the basic idea of our heuristic.

\section{Insert Figure 2}

\subsection{Formal description of the heuristic}

In the following we formalize the proposed heuristic to solve the LB-ALBP.

Given a problem $P R^{0}$, it is defined by the following attributes:

- $\quad P R^{0} . N$, the set of tasks.

- $\quad P R^{0} . t_{i}$, the processing time of task $i\left(i \in P R^{0} . N\right)$.

- $\quad P R^{0} . P$, the set of couples of tasks $\left(i, i^{\prime}\right)$ where $i$ is immediate predecessor of $i^{\prime}$.

- $\quad P R^{0} . m$, the number of workstations.

On the other hand, a solution consists of an assignment $S T[i] \in\left\{1, \ldots, P R^{0} . m\right\}$ for task $i$ $\left(i \in P R^{0} . N\right)$, i.e. $S T[i]$ is the station in which task $i$ is assigned.

The algorithm of the designed heuristic is shown in Figure 3. The algorithms of the functions Solve_SALBP-2 (Steps 0 and 9), Get_subproblem_left (Step 6) and Get_subproblem_right (Step 6) are explained in Subsections 2.2.1, 2.2.2 and 2.2.3, respectively. With respect to the local search (Steps 1 and 11), different variants can be obtained; we have proposed 13 variants which are explained in Section 2.3.

\section{Insert Figure 3}

The heuristic starts obtaining an initial solution by solving the $P R^{0}$ problem as a SALBP-2 (Step 0). Then it applies a local search to the obtained solution and the best solution obtained up to now is initialized with the found local optimum (Step 1). The current subproblem and its current solution are initialized as the global ones (Step 2) and the set with the remaining subproblems to solve is initialized with a void set (Step 3). The variable $i d \_f s t$ is used to know the original numeric identification of the first station of the current subproblem; at the first iteration, since the current subproblem is $P R^{0}, i d \_f s t$ is initialized to 1 (Step 4). The variable $i d \_s t$ is used when a subproblem $P R^{\prime}$ is solved and its solution $S T$ ' is obtained with $P R^{\prime} . m$ 
workstations $\left(j=1, \ldots, P R^{\prime} . m\right)$ : we can update the global solution $S T$ knowing that the station $j$ of $S T$ ' corresponds to the station $j+i d \_f s t-1$ of $S T$; for example, the $i d \_f s t$ of Subproblem 2 shown in Figure $2 \mathrm{a}$ is equal to 5 , the subproblem is solved with 10 stations $(j=1, \ldots, 10)$ and the stations $5(1+5-1)$ to $14(10+5-1)$ of the global solution are updated. Then the most workloaded station (if there is more than one most workloaded station, then the most central is chosen) is used as a reference to split the problem into zero, one or two subproblems (Steps 5-6): one with the tasks that are in the stations at the left of the most workloaded station (if there are stations at the left), and another subproblem with the tasks that are in the stations at the right of the most workloaded station (if there are stations at the right). The generated subproblems are solved as SALBP-2 (Step 9), too. The criterion for choosing the next subproblem to solve is the subproblem whose solution has the most workloaded station (to break the tie, the oldest generated subproblem is chosen) and it is removed from the remaining subproblems to solve (Step 8). The solution obtained of this subproblem is used to update the global solution (Step 10). Each time that the global solution is updated, a local search is applied. In the case that the obtained local optimum is better than the best solution found up to now, the process is reset but starting from this local optimum (Step 11). The heuristic ends when no subproblems remain for being solved (Step 7).

\subsubsection{Solving SALBP-2}

The procedure of the solution of the SALBP-2 problem is based on solving iteratively the SALBP-1 problem. This way of solving the SALBP-2 is usual in the literature (e.g., Pastor et al., 2002) and it consists of the following steps:

Step 1. Calculating the lower bound on the cycle time, $C T: C T=\max \left\{t_{\max },\left\lceil\frac{T}{m}\right\rceil\right\}$, where $t_{\max }$ is the maximum processing time of all tasks, $T$ is the sum of the processing times of all tasks, $m$ is the number of stations and $\lceil x\rceil$ the operator that returns the smallest integer that is equal to or greater than $x$.

Step 2. Solving the instance by an heuristic for the SALBP-1 and using a cycle time equal to $C T$.

Step 3. If the number of stations of the obtained solution is equal to $m$, then the procedure ends (and returns the last obtained solution); otherwise, $C T=C T+1$ and go to Step 2.

Note that the increment of the cycle time by 1 unit and using $\lceil T / \mathrm{m}\rceil$ instead of $T / \mathrm{m}$ are possible because, without losing generality, we can assume that the processing times of the tasks are integers.

12 different well-known heuristics for the SALBP-1 are used when solving the (sub)problems as SALBP-2 in our proposed heuristic. The solution returned to our heuristic is the best one (according to the LB-ALBP criterion) of the 12 obtained solutions.

All 12 heuristics for the SALBP-1 are based on the same greedy procedure and the only difference between them is the priority rule that is used to select the next task to be assigned (these 12 priority rules are explained in the Appendix). The greedy procedure is iterative and workstation-oriented; i.e., at each iteration the best candidate task (according to the priority 
rule) is assigned to the station $j$ which is being completed (as mentioned in Andrés et al. (2008), most computational experiments reported in the literature indicate that, for SALBP, workstation-oriented procedures provide better results than task-oriented ones, although they are not theoretically dominant (Scholl and Voß, 1996)). A task $i$ is candidate if all its precedent tasks have been already assigned and it fits in station $j$. If there are not available candidate tasks (but there are still tasks to be assigned) then station $j$ is closed and the next station $j+1$ is opened. The procedure ends when all tasks have been assigned.

\subsubsection{Generating the left subproblem}

The algorithm of the generation of the left subproblem, which concerns to the function Get_subproblem_left $\left(P R^{\prime}, S T^{\prime}, k\right)$, is shown in Figure 4.

\section{Insert Figure 4}

\subsubsection{Generating the right subproblem}

The algorithm of the generation of the right subproblem, which concerns to the function Get_subproblem_right $\left(P R^{\prime}, S T^{\prime}, k\right)$, is shown in Figure 5.

\section{Insert Figure 5}

\subsection{Local search procedures}

The local search procedures are used at each iteration of the proposed heuristic with the aim of improving the obtained global solution. We finally have tested 13 local search procedures which are based on generating and exploring the neighbourhood of the current solution. The neighbourhood of a solution is made with the set of its neighbouring solutions which are obtained by applying a transformation rule to the current solution. The three following transformation rules have been applied in this work:

Neighbourhood 1 (N1). This transformation rule is based on the local optimization method known as trade and transfer of elements. Let $k$ and $p$ be the stations that will take part in the trade and transfer of tasks, where the workload of the station $k, W L[k]$, is greater than the workload of station $p, W L[p]$. The neighbourhood of the current solution is made up of the solutions that are obtained by generating all feasible trades (i.e., satisfying the precedence relations) between the tasks of station $k$ and the tasks of station $p$, and next generating all feasible transfers of the tasks of station $k$ to station $p$.

Neighbourhood 2 (N2). Given station $k$, the neighbourhood of the current solution is made up of the solutions that are obtained by generating all feasible trades and transfers between the tasks of the station $k$ and the others stations.

Neighbourhood 3 (N3). The neighbourhood of the current solution is made up of the solutions that are obtained by generating all feasible trades and transfers between the tasks of all pairs of stations.

The two proposed generation and exploration of the neighbourhood are as follows: 
First improvement strategy (FIS). Given a current solution $S T$, one neighbour nst is generated. If $n s t$ is better (according to the LB-ALBP criterion) than $S T$, then $S T=n s t$ and the procedure is reset; otherwise, the next neighbour of $S T$ is generated. The procedure finishes when all neighbours of the current solution $S T$ have been generated and none is better than $S T$.

Best improvement strategy (BIS). Given a current solution $S T$, all neighbours are generated and the best one, $n s t$, is chosen. If $n s t$ is better than $S T$, then $S T=n s t$ and the procedure is reset; otherwise, the procedure finishes.

The 13 tested local search procedures are explained in the following subsections (2.3.1. to 2.3.4.).

\subsubsection{Local Search Procedures LSP1, LSP2 and LSP3}

The main characteristics of this first local search procedure, LSP1, are the use of the transformation rule Nl and the use of the first improvement strategy, FIS. This local search procedure is shown in Figure 6.

\section{Insert Figure 6}

As we can see in Figure 6, LSP1 is based on exploring the neighbourhood obtained by using the lowest loaded station and the most loaded one, then by using the lowest and second most loaded stations and so on until using the lowest and second lowest loaded stations; then, the same mechanism is followed by using the second lowest loaded station and the other ones (most loaded, second most loaded, ...), and so on.

The second local search procedure, $L S P 2$, is very similar to $L S P 1$ except for the Step 3, in which the exploration BIS is used instead of FIS.

The main characteristics of the third local search procedure, LSP3, are the use of the transformation rule $N 2$ and the use of the best improvement strategy, BIS. It is based on exploring the neighbourhood obtained by using the lowest loaded station, then by using the second lowest loaded stations and so on.

\subsubsection{Local Search Procedures $L S P a, L S P b$ and, $L S P c$}

The local search procedures $L S P a, L S P b$ and $L S P c$ can be seen as variants of $L S P 1, L S P 2$ and $L S P 3$, respectively, in which the order of the iterations has been inverted. For instance, $L S P a$ use the transformation rule $N I$ and the first improvement strategy, FIS, but it is based on exploring the neighbourhood obtained by using the most loaded station and the lowest loaded one, then by using the most loaded and the second lowest loaded stations and so on until using the most loaded and second most loaded stations; then, the same mechanism is followed by using the second most loaded station and the other ones (lowest loaded, second lowest loaded, ...), and so on.

\subsubsection{Local Search Procedures $L S P 1+a, L S P 2+b, L S P 3+c, L S P a+1, L S P b+2$ and $L S P c+3$}




\section{Computational experiment}

In this work, 13 variants of a new heuristic procedure for solving the LB-ALBP have been developed. The variants are due to the local search procedure used in the designed heuristic. In the remaining of this paper, we will name them as V-LSP1, V-LSP2, V-LSP3,V-LSPa, V$L S P b, V-L S P c, V-L S P 1+a, V-L S P 2+b, V-L S P 3+c, V-L S P a+1, V-L S P b+2, V-L S P c+3$ and $V-$ $L S P$ all in which the local search procedures described in Sections 2.3.1 to 2.3.4 are used, respectively.

Moreover, for comparison purpose, the two following procedures are also used to solve the LB-ALBP:

- To solve the LB-ALBP as a SALBP-2 using the 12 rules shown in the Appendix as it is explained in Section 2.2.1 and get the best solution; let it be named $B H$. Notice that $B H$ returns the initial solution used in the designed variants.

- To apply our proposed heuristic to solve the LB-ALBP without applying any local search procedure (neither Steps 1 nor 11 of Figure 3); let it be named $V$-noLSP.

All algorithms were coded in Java and the computational experiment was carried out using a 3.0 GHz Pentium IV with $1 \mathrm{~GB}$ of RAM (the same computer which was used in Pastor (2011) to test H1_SHM, the best heuristic procedure published to date).

\subsection{Instances for the computational experiment}

The effectiveness of the 13 heuristic variants designed for solving the LB-ALBP has been evaluated with the 17 well-known test problems available at the Scholl and Klein's assembly line balancing research website (www.assembly-line-balancing.de), from which 302 test instances are obtained. We observed that the solution of the problem called Tonge with $m=$ 25 returned, when it is solved as a SALBP-2, 24 stations; thus, we removed it from the test instances and we finally worked with 301 test instances. Table 1 lists each problem with its 
respective name; number of tasks $(N)$; minimum, maximum and average processing times of the tasks $\left(t_{\min }, t_{\max }\right.$ and $\bar{t}$, respectively); order strength of the precedence graph $(O S)$; and the range of the number of workstations of its instances ( $m$-Range).

\section{Insert Table 1}

\subsection{Results of the computational experiment}

First the 13 variants of the proposed heuristic, together with $B H$ and $V-n o L S P$, are compared with $H 1 \_S H M$, the best heuristic procedure published for solving the LB-ALBP (Section 3.2.1). Next the best variants are compared between them in order to establish which one returns, on average, the best results taking into account the influence of the characteristics of the instances (Section 3.2.2). After that (Section 3.2.3), the best variants are applied to the instances that are solved optimally in Pastor (2011). Finally, other strategies for the local search are tried (Section 3.2.4).

We use the parameter $\delta$ to compare between solutions. Note that the worse a solution with respect to the reference solution, the larger the value of $\delta$.

\subsubsection{Comparison versus the best published heuristic procedure}

The best heuristic procedure specifically designed to solve the LB-ALBP has been proposed in Pastor (2011), called H1_SHM by the author, which is based on MILP. H1_SHM consists of carrying out a preemptive goal programming for 18,000 seconds in total; i.e., it sequentially solves $m-1$ (MILP) models by observing and sequentially minimising the objectives once the optimal values of the highest-priority objectives have been obtained. The first submodel is run with a computing time limit of 18,000 seconds; if any time remains available, the second submodel is run with a time limit equal to the remaining computing time; and so on.

We compare the values of $\delta$ obtained by all algorithms with an ANOVA analysis; the $\delta$ values are calculated, for each instance, by comparing each solution with the best solution obtained using the 16 algorithms. Figure 7 summarises the main conclusions obtained by means of the Fisher Test Graphics provided by ANOVA. We can observe that the quality of the solutions obtained by $H 1 \_S H M$ is clearly worse, on average, than the quality of the solutions obtained by all variants of the new heuristic. These first results would be expected because H1_SHM is basically the running of sequential MILP problems within a time limit whereas the algorithms proposed in this work are adhoc heuristic methods for the LB-ALBP. On the other hand, as it is also expected: i) all variants of our proposed heuristic are able to improve considerably the solutions generated by $B H$; and ii) the addition of a local search procedure is crucial for obtaining much better solutions.

\section{Insert Figure 7}

To do a more in-depth analysis, additional information is shown in Table 2: minimum, maximum and average values of the metric $\delta\left(\delta_{\min }, \delta_{\max }\right.$ and $\bar{\delta}$, respectively); the standard deviation value of $\delta\left(\sigma_{\delta}\right)$; and the minimum, maximum and average processing time $\left(t_{\min }, t_{\max }\right.$ and $\bar{t}$, respectively), in seconds, of the procedures. We can see that the performance of 
$H 1 \_S H M$ is worse than the performance of the other procedures: not only it gives worse values of $\delta$ but, moreover, it has by far the greatest average $\sigma_{\delta}$ dispersion and its average processing time is much larger. According to the local search used, different performances are obtained. The best $\delta$ average is obtained with $V-L S P a$, which also has the least variability in the $\delta$ values of its solutions, and the worst $\delta$ average is obtained with $V$-LSPall. With respect to the average processing time, all 13 proposed variants have an average between 50 and 74 seconds.

\section{Insert Table 2}

Anyway, all variants have a similar performance with respect to their $\delta$ average, especially if we compare them with $H 1 \_S H M, B H$ and $V$-noLSP. However, a more in-depth analysis given in the next section will show that different performances are obtained according to the characteristics of the instance to solve.

\subsubsection{Comparison between the proposed variants of the heuristic}

In this section we analyse the influence of the characteristics of the instances -in particular, order strength $O S$ (which gives information on the complexity of the precedence graph) and number of tasks $N$ (which indicates the size of the instance)- on the quality of the obtained solutions. The instances have been classified according to $O S$ and $N$ as follows: (i) Low-OS $(22.67 \leq O S \leq 25.80)$, Middle-OS $(40.38 \leq O S \leq 59.50)$ and High-OS $(77.55 \leq O S \leq 83.82)$; (ii) Low-N $(29 \leq N \leq 35)$, Middle- $N(45 \leq N \leq 111)$ and High- $N(148 \leq N \leq 297)$.

To facilitate the presentation of the results, we will focus into the 5 best variants according to the different characteristics of the instances, which are $V-L S P 1, V-L S P a, V-L S P c, V-L S P 1+a$ and $V-L S P a+1$. Note that the $\delta$ values are calculated, for each instance, by comparing each solution with the best solution obtained using the aforementioned 5 best variants. As we can see in Figure 8a, the best $\delta$ averages for the High-OS, Middle-OS and Low-OS instances are obtained with $V$-LSPI $+a, V-L S P I$ and $V-L S P a$, respectively. With respect to the number of tasks, Figure $8 \mathrm{~b}$ shows that the best $\delta$ averages for the High-N, Middle- $N$ and Low-N instances are obtained with $V-L S P a+1, V-L S P a$ and $V-L S P 1$, respectively.

\section{Insert Figure 8}

An analysis of the existing interactions among the considered characteristics (order strength and number of tasks, $O S / N$ ) is shown in Table 3 (together with the number of instances of each class, in parentheses). We can see that $H / L$ instances are solved similarly by the best variants independently of the $O S$ of the instance and for the $M / L$ instances the best variant is $V$-LSP1. For $L o w-O S$ instances $(L / M$ and $L / H)$, the best procedure variant depends on their number of tasks: if their $N$ is middle, then it is clear that $V-L S P a$ is the best variant; on the other hand, if their $N$ is high, then $V-L S P a+1$ obtains the best $\delta$ average. On the other hand, $V$ $L S P I+a$ is the best procedure for solving $H / M$ instances, and also for solving $M / M$ instances together with $V$ - $L S P 1$. Finally, $V$ - $L S P c$ is the best procedure for solving $M / H$ instances.

\section{Insert Table 3}

\subsubsection{Comparison versus optimal solutions}


62 optimal solutions of the test instances were obtained in Pastor (2011) by means of MILP. For one of these instances (Arcus2 with 3 stations) the optimization software guaranteed the optimal solution, although this solution is not optimal (our proposed procedure has found a better solution). This is due to the allowed relative MIP gap tolerance, since the cycle time in this instance is very high. Thus, we compare the solutions returned by our procedure variants with the other 61 solutions. Table 4 shows the percentage of instances in which the optimal solution is found (\%opt), the minimum, maximum, average and standard deviation values of the metric $\delta\left(\delta_{\min }, \delta_{\max }, \bar{\delta}\right.$ and $\sigma_{\delta}$, respectively); and the minimum, maximum and average processing time $\left(t_{\min }, t_{\max }\right.$ and $\bar{t}$, respectively), in seconds, of the procedures. The $\delta$ values are calculated, for each instance, by comparing each solution with the optimal solution.

\section{Insert Table 4}

Around more of the $25 \%$ of the solutions returned by the best variants of our procedure are optimal. With respect to the other solutions, we can see that they are not far from the optimal ones and using a computing time, on average, around 1,000 times lower than the MILP model. We should also note that the performances of all variants are robust since the average $\sigma_{\delta}$ dispersion is low.

\subsubsection{Other strategies for the local search}

To evaluate de benefits of solution and local search procedures not only the quality of the obtained solutions should be taken into account but also the computing time needed to obtain them. For instance, it is well known that MILP models and B\&B algorithms always ensures an optimal solution but the computing time may be astronomical.

The computational experiment shows the following: i) the algorithms that we propose obtains better solutions, on average, than the solutions existing in the literature, and ii) they use a little fraction of the computing time needed to obtain these previous solutions. But the use of more complex neighbourhoods could improve the quality of the solutions. We tested two other kinds of movements:

Trade between two pairs of tasks and transfer of a pair of tasks. The obtained solutions are always equal to or worse than the solutions obtained with the original local search procedures and, moreover, the computing time is increased around 3 times.

Trade and transfer allowing non-improving search strategy. When there is not a better neighbour, it is allowed to jump to a non-worse neighbour that has not been visited. We tested this kind of strategy in the algorithm that provides the better $\delta$ average, $V-L S P a$, and the solutions, on average, hardly improves at the expense of increasing the computing time around 150 times (on average, 8,499.67 seconds). The $\delta$ average (calculated with respect to the lower bound proposed in Pastor (2011)) obtained by allowing non-improving movements is only $1.79 \%$ better than the $\delta$ average obtained without allowing them. Thus, the trade-off between the improvement of the solutions and the extra computing time seems not good and we think that a better trade-off could be obtained using more elaborated metaheuristic procedures such as simulated annealing, tabu search or variable neighbourhood search. We propose this issue as future research in Section 4. 
From a practical point of view, when a novel LB-ALBP case has to be solved in a real implementation, the available time and other sources, such as number and type of computers, will determine the actions for obtaining a solution for the company. The analysis of section 3.2.2 shows which variant of our heuristic is the best, on average, to solve a case according to its characteristics. However, the decision maker should decide which procedures will be run according to the particular real-life context in which the problem is solved.

\section{Conclusions}

In this paper we propose and test a new heuristic procedure for solving the Lexicographic Bottleneck Assembly Line Balancing Problem (LB-ALBP), a new assembly line balancing problem, which hierarchically minimizes the workload of the most heavily loaded workstation, followed by the workload of the second most heavily loaded workstation, followed by the workload of the third most heavily loaded workstation, and so on.

The proposed procedure combines a heuristic procedure for obtaining an initial solution and several local optimization procedures. The heuristic is based on splitting iteratively subproblems into smaller subproblems (i.e., subproblems with fewer stations and fewer tasks to be assigned) and solving each of them as a SALBP-2; the obtained solution of each subproblem is used to update the global solution of the original problem; and, moreover, a local search is applied at each iteration of the heuristic.

The computational experiment shows that the new heuristic procedure is an improvement upon the heuristic procedures published to date (three heuristic procedures based on two mixed-integer linear programming models). An additional comparison versus optimal solutions shows that an extra effort should be done to design more powerful heuristic procedures. Thus, future research work will involve designing and analyzing more powerful heuristics (for example, for taking advantage of the proposed heuristic we could replace the classical local search procedures by more sophisticated improvement procedures as tabu search, simulated annealing or variable neighbourhood search metaheuristics) and/or pure metaheuristic methods for solving the LB-ALBP.

\section{Appendix}

The 12 used priority rules are shown in Table 5 (Helgeson and Birnie (1961), Tonge (1961), Arcus (1963), Moodie and Young (1965) and Talbot and Patterson (1984)), where $C T$ is the cycle time, $n$ the number of tasks, $N S_{i}$ the total number of tasks which succeed (precede) task $i, N I S_{i}$ the number of tasks which must immediately succeed task $i, S_{i}\left(P_{i}\right)$ the set of tasks which must succeed (precede) task $i$, and $t_{i}$ the processing time of task $i$.

\section{Insert Table 5}

\section{References}


Ağpak, K., Gökçen, H., 2005. Assembly line balancing: Two resource constrained cases. International Journal of Production Economics, 96 (1), 129-140.

Amen, M., 2006. Cost-oriented assembly line balancing: Model formulations, solution difficulty, upper and lower bounds. European Journal of Operational Research, 168 (3), 747-770.

Andrés, C., Miralles, C., Pastor, R., 2008. Balancing and scheduling tasks in assembly lines with sequence-dependent setup times. European Journal of Operational Research, 187 (3), 1212-1223.

Arcus, A.L., 1963. An analysis of a computer method of sequencing assembly line operations. Ph.D. dissertation, University of California, Berkeley.

Baybars, I., 1986. A survey of exact algorithms for the simple assembly line balancing problem. Management Science, 32 (8), 909-932.

Becker, C., Scholl, A., 2006. A survey on problems and methods in generalized assembly line balancing. European Journal of Operational Research, 168 (3), 694-715.

Berman, O., Ingco, D.I., Odoni, A., 1994. Improving the location of minimax facilities through network modification. Networks, 24 (1), 31-41.

Boysen, N., Fliedner, M., Scholl, A., 2006. A classification of assembly line balancing problems. Working paper 12/2006, Friedrich-Schiller-Universität Jena: Thuringia, Germany.

Boysen, N., Fliedner, M., Scholl, A., 2007. A classification of assembly line balancing problems. European Journal of Operational Research, 183 (2), 674-693.

Boysen, N., Fliedner, M., Scholl, A., 2008. Assembly line balancing: Which model to use when? International Journal of Production Economics, 111 (2), 509-528.

Burkard, R.E., Rendl, F., 1991. Lexicographic bottleneck problems. Operations Research Letters, 10 (5), 303-308.

Capacho, L., Pastor, R., Dolgui, A., Gunshinskaya, O. 2009. An evaluation of constructive heuristic methods for solving the alternative subgraphs assembly line balancing problem. Journal of Heuristics, 15 (2), 109-132.

Corominas, A., Pastor, R., Plans, J., 2008. Balancing assembly line with skilled and unskilled workers. OMEGA, 36 (6), 1126-1132.

Corominas, A., Pastor, R., 2009. A note on "A comparative evaluation of assembly line balancing heuristics". International Journal of Advanced Manufacturing Technology, 44 (7-8), 817.

Corominas, A., Ferrer, L., Pastor, R., 2010. "Assembly line balancing: general resourceconstrained case". International Journal of Production Research. doi: 10.1080/00207543.2010.481294.

Cortés, P., Muñuzuri, J., Onieva, L., Larrañeta, J., Vozmediano J.M., Alarcón, J.C., 2006. Andalucía assesses the investment needed to deploy a fiber-optic network. Interfaces, 36 (2), 105-117.

Cortés, P., Onieva, L., Guadix, J., 2010. Optimising and simulating the assembly line balancing problem in a motorcycle manufacturing company: a case study. International Journal of Production Research, 48 (12), 3637-3656.

Ding, F-Y., Zhu, J., Sun, H., 2006. Comparing two weighted approaches for sequencing mixed-model assembly lines with multiple objectives. International Journal of Production Economics, 102 (1), 108-131.

Gamberini, R., Grassi, A., Rimini, B., 2006. A new multi-objective heuristic algorithm for solving the stochastic assembly line re-balancing problem. International Journal of Production Economics, 102 (2), 226-243. 
Gökçen, H., Ağpak, K., Benzer, R., 2006. Balancing of parallel assembly lines. International Journal of Production Economics, 103 (2), 600-609.

Helgeson, W.B., Birnie, D.P., 1961. Assembly line balancing using Ranked Positional Weight Techniques. Journal of Industrial Engineering, 12 (6), 394-398.

Hoffman, T.R., 1992. EUREKA: a hybrid system for assembly line balancing. Management Science, 38 (1), 39-47.

Johnson, R.V., 1988. Optimally balancing large assembly lines with Fable. Management Science, 34 (2), 240-253.

Kao, E.P.C., Queyranne, M., 1982. On dynamic programming methods for assembly line balancing. Operations Research, 30 (2), 375-390.

Krajewski, L.J., Ritzman, L.P., 1979. Disaggregation in manufacturing and service organizations: Survey of problems and research, en Ritzman, L.P., Krajewski, L.J., Berry, W.L., Goodman, S.H., Hardy, S.T. and Vitt, L.D Eds. Disaggregation. Problems in Manufacturing and Service Organizations, Boston, MA: Martinus Nijholff Publishing.

Martino, L., Pastor, R., 2010. Heuristic procedures for solving the general assembly line balancing problem with setups. International Journal of Production Research, 48 (6), 1787-1804.

Moodie, C.L., Young, H.H., 1965. A heuristic method of assembly line balancing for assumptions of constant or variable work element times. Journal of Industrial Engineering, 16 (1), 23-29.

Park, K., Park, S., Kim, W., 1997. A heuristic for an assembly line balancing problem with incompatibility, range, and partial precedence constraints. Computers \& Industrial Engineering, 32 (2), 321-332.

Pastor, R., Andrés, C., Duran, A., Pérez, M., 2002. Tabu search algorithms for an industrial multi-product and multi-objective assembly line balancing problem, with reduction of the tasks dispersion. Journal of the Operational Research Society, 53 (12), 1317-1323.

Pastor, R., Ferrer, L., 2009. An improved mathematical program to solve the simple assembly line balancing problem. International Journal of Production Research, 47 (11), 29432959.

Pastor, R., 2011. LB-ALBP: the lexicographic bottleneck assembly line balancing problem. International Journal of Production Research, 49 (8), 2425-2442.

Pentico, D.W., 2007. Assignment problems: A golden anniversary survey. European Journal of Operational Research, 176 (2), 774-793.

Ponnambalam, S.G., Aravindan, P., Mogileeswar, G., 1999. A comparative evaluation of assembly line balancing heuristics. International Journal of Advanced Manufacturing Technology, 15 (8), 577-586.

Rekiek, B., Dolgui, A., Delchambre, A., Bratcu, A., 2002. State of art of optimization methods for assembly line design, Annual Reviews in Control, 26 (2), 163-174.

Scholl, A., Voß, S., 1996. Simple assembly line balancing-Heuristic approaches. Journal of Heuristics, 2, 217-244.

Scholl, A., Klein, R., 1997. SALOME: a bidirectional branch and bound procedure for assembly line balancing. Informs Journal on Computing, 9 (4), 319-334.

Scholl, A., Becker, C., 2006. State-of-the-art exact and heuristic solution procedures for simple assembly line balancing. European Journal of Operational Research, 168 (3), 666693.

Sokkalingam, P.T., Aneja, Y.P., 1998. Lexicographic bottleneck combinatorial problems. Operations Research Letters, 23 (1-2), 27-33. 
Talbot, F.B., Patterson, J.H., 1984. An integer programming algorithm with network cuts for solving the assembly line balancing problem. Management Science, 30 (1), 85-99.

Talbot, F., Patterson, J.H., Gehrlein, W.V., 1986. A comparative evaluation of heuristic line balancing techniques. Management Science, 32 (4), 431-453.

Tonge, F.M., 1961. A heuristic program for assembly line balancing. Prentice-Hall, Englewood Cliffs, NJ.

White, W.W., 1961. Comments on a paper by Bowman. Operations Research, 9 (2), 274-276.

Yu, G., 1998. Min-Max optimization of several classical discrete optimization problems. Journal of Optimization Theory and Applications, 98 (1), 221-242. 


\begin{tabular}{|l|c|c|c|c|l|c|}
\hline Name & $N$ & $t_{\min }$ & $t_{\max }$ & $\bar{t}$ & OS & m-Range \\
\hline Arcus1 & 83 & 233 & 3691 & 912.1 & 59.09 & 3 to 22 \\
\hline Arcus2 & 111 & 10 & 5689 & 1354.9 & 40.38 & 3 to 27 \\
\hline Barthold & 148 & 3 & 383 & 38.1 & 25.80 & 3 to 15 \\
\hline Barthol2 & 148 & 1 & 83 & 28.6 & 25.80 & 27 to 51 \\
\hline Buxey & 29 & 1 & 25 & 11.2 & 50.74 & 7 to 14 \\
\hline Gunther & 35 & 1 & 40 & 13.8 & 59.50 & 6 to 15 \\
\hline Hahn & 53 & 40 & 1775 & 264.6 & 83.82 & 3 to 10 \\
\hline Killbridge & 45 & 3 & 55 & 12.3 & 44.55 & 3 to 11 \\
\hline Lutz1 & 32 & 100 & 1400 & 441.9 & 83.47 & 8 to 12 \\
\hline Lutz2 & 89 & 1 & 10 & 5.4 & 77.55 & 9 to 28 \\
\hline Lutz3 & 89 & 1 & 74 & 18.5 & 77.55 & 3 to 23 \\
\hline Mukherje & 94 & 8 & 171 & 44.8 & 44.80 & 3 to 26 \\
\hline Sawyer & 30 & 1 & 25 & 10.8 & 44.83 & 7 to 14 \\
\hline Scholl & 297 & 5 & 1386 & 234.5 & 58.16 & 25 to 52 \\
\hline Tonge & 70 & 1 & 156 & 50.1 & 59.42 & 3 to 24 \\
\hline Warnecke & 58 & 7 & 53 & 26.7 & 59.10 & 3 to 29 \\
\hline Wee-Mag & 75 & 2 & 27 & 20.0 & 22.67 & 3 to 30 \\
\hline
\end{tabular}

Table 1. Test problems from the Scholl and Klein's website

\begin{tabular}{|c|c|c|c|c|c|c|c|}
\hline & $\delta_{\min }$ & $\delta_{\max }$ & $\bar{\delta}$ & $\sigma_{\delta}$ & $t_{\min }$ & $t_{\max }$ & $\bar{t}$ \\
\hline H1_SHM & 0 & 26.2386 & 1.0936 & 3.1219 & 0.00 & $18,000.00$ & $14,725.63$ \\
\hline$B H$ & 0 & 5.0510 & 0.9155 & 1.1126 & 0.00 & 44.28 & 4.55 \\
\hline$V$-noLSP & 0 & 5.0510 & 0.9125 & 1.1109 & 0.00 & $4,219.25$ & 62.48 \\
\hline$V-L S P 1$ & 0 & 5.0500 & 0.6785 & 1.0341 & 0.00 & $3,226.49$ & 67.24 \\
\hline$V-L S P 2$ & 0 & 5.0500 & 0.6745 & 1.0564 & 0.00 & $3,039.04$ & 56.24 \\
\hline$V-L S P 3$ & 0 & 5.0500 & 0.6838 & 1.0432 & 0.00 & $2,237.24$ & 50.03 \\
\hline$V-L S P a$ & 0 & 5.0500 & 0.6456 & 1.0110 & 0.00 & $2,951.96$ & 57.62 \\
\hline$V-L S P b$ & 0 & 5.0500 & 0.6690 & 1.0368 & 0.00 & $2,731.54$ & 54.69 \\
\hline$V-L S P c$ & 0 & 5.0500 & 0.6850 & 1.0210 & 0.00 & $3,327.71$ & 73.66 \\
\hline$V-L S P I+a$ & 0 & 5.0500 & 0.6537 & 1.0130 & 0.02 & $2,504.53$ & 64.13 \\
\hline$V-L S P 2+b$ & 0 & 5.0500 & 0.6726 & 1.0330 & 0.02 & $2,641.86$ & 64.77 \\
\hline$V-L S P 3+c$ & 0 & 5.0500 & 0.6752 & 1.0573 & 0.02 & $2,514.39$ & 55.96 \\
\hline$V-L S P a+1$ & 0 & 5.0500 & 0.6646 & 1.0384 & 0.02 & $3,277.14$ & 61.45 \\
\hline$V-L S P b+2$ & 0 & 5.0500 & 0.6739 & 1.0241 & 0.02 & $3,193.36$ & 58.92 \\
\hline$V-L S P c+3$ & 0 & 5.0500 & 0.6752 & 1.0205 & 0.02 & $3,104.73$ & 69.71 \\
\hline$V$-LSPall & 0 & 5.0500 & 0.6919 & 1.0385 & 0.02 & $3,727.22$ & 66.44 \\
\hline
\end{tabular}

Table 2. Results obtained with the 16 algorithms 


\begin{tabular}{|l|r|r|c|r|r|c|r|r|}
\cline { 2 - 9 } \multicolumn{1}{c|}{} & \% opt & $\delta_{\min }$ & $\delta_{\max }$ & $\bar{\delta}$ & \multicolumn{1}{c|}{$\sigma_{\delta}}$, & $t_{\min }$ & \multicolumn{1}{c|}{$t_{\max }$} & \multicolumn{1}{c|}{$\bar{t}$} \\
\hline$M I L P$ & 100.00 & 0 & 0 & 0 & 0 & 0.00 & $17,712.21$ & 998.83 \\
\hline$V-L S P 1$ & 24.59 & 0 & 2.4390 & 0.4378 & 0.6809 & 0.00 & 8.92 & 1.10 \\
\hline$V-L S P a$ & 29.51 & 0 & 2.4390 & 0.4377 & 0.6816 & 0.00 & 6.56 & 0.86 \\
\hline$V-L S P c$ & 27.87 & 0 & 2.4390 & 0.4345 & 0.6823 & 0.02 & 11.73 & 1.18 \\
\hline$V-L S P 1+a$ & 27.87 & 0 & 2.4390 & 0.4386 & 0.6815 & 0.02 & 8.12 & 1.14 \\
\hline$V-L S P a+1$ & 27.87 & 0 & 2.4390 & 0.4407 & 0.6805 & 0.02 & 8.04 & 0.82 \\
\hline
\end{tabular}

Table 4. Results obtained for the 61 instances whose optimal solution is known

\begin{tabular}{|l|l|l|c|}
\hline $\begin{array}{l}\text { Maximum ranked positional } \\
\text { weight }\end{array}$ & $R P W_{i}=t_{i}+\sum_{i^{\prime} \in S_{i}} t_{i^{\prime}}$ & Minimum slack & $U B_{i}-L B_{i}$ \\
\hline Maximum task time & $t_{i}$ & Minimum task number & $i$ \\
\hline $\begin{array}{l}\text { Maximum total number of } \\
\text { follower tasks }\end{array}$ & $N S_{i}$ & $\begin{array}{l}\text { Maximum average ranked } \\
\text { positional weight }\end{array}$ & $\frac{R P W_{i}}{N S_{i}+1}$ \\
\hline $\begin{array}{l}\text { Maximum number of } \\
\text { immediate follower tasks }\end{array}$ & $N I S_{i}$ & $\begin{array}{l}\text { Minimum upper bound } \\
\text { divided by followers }\end{array}$ & $\frac{U B_{i}}{N S_{i}+1}$ \\
\hline $\begin{array}{l}\text { Minimum lower bound } \\
\text { Minimum upper bound }\end{array}$ & $L B_{i}=\left[\frac{t_{i}+\sum_{i^{\prime} \in P i} t_{i^{\prime}}}{C T}\right]$ & $\begin{array}{l}\text { Maximum task time divided } \\
\text { by upper bound }\end{array}$ & $\frac{t_{i}}{U B_{i}}$ \\
\hline
\end{tabular}

Table 5. Priority rules used by the greedy procedure for solving the SALBP-1 
1

2

3

4

5

6

7

8

9

FIGURE CAPTION

Figure 1. Two possible workload distributions

Figure 2. Example of generation of subproblems

Figure 3. Algorithm of the proposed heuristic

Figure 4. Algorithm of the function Get_subproblem_left $\left(P R^{\prime}, S T^{\prime}, k\right)$

Figure 5. Algorithm of the function Get_subproblem_right $\left(P R^{\prime}, S T^{\prime}, k\right)$

Figure 6. Local search procedure $L S P 1$

Figure 7. Delta means and 95\% LSD Intervals Graphic for the 16 algorithms

Figure 8. Interaction plot for the characteristics of the instances 

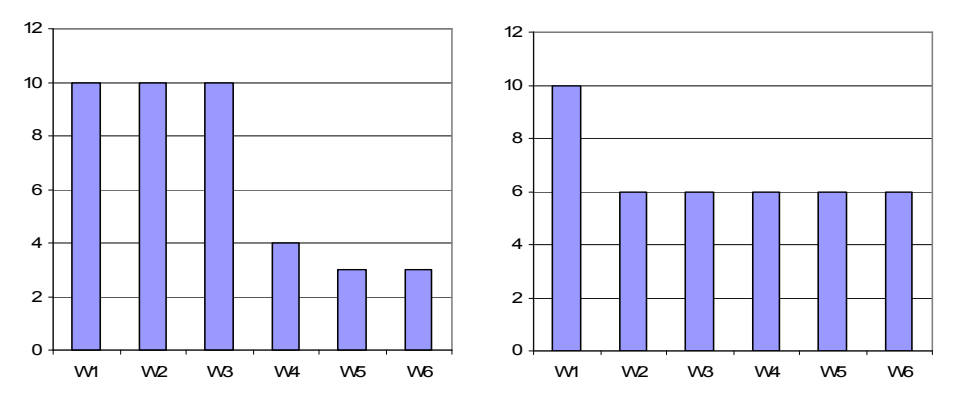

Figure 1. Two possible workload distributions

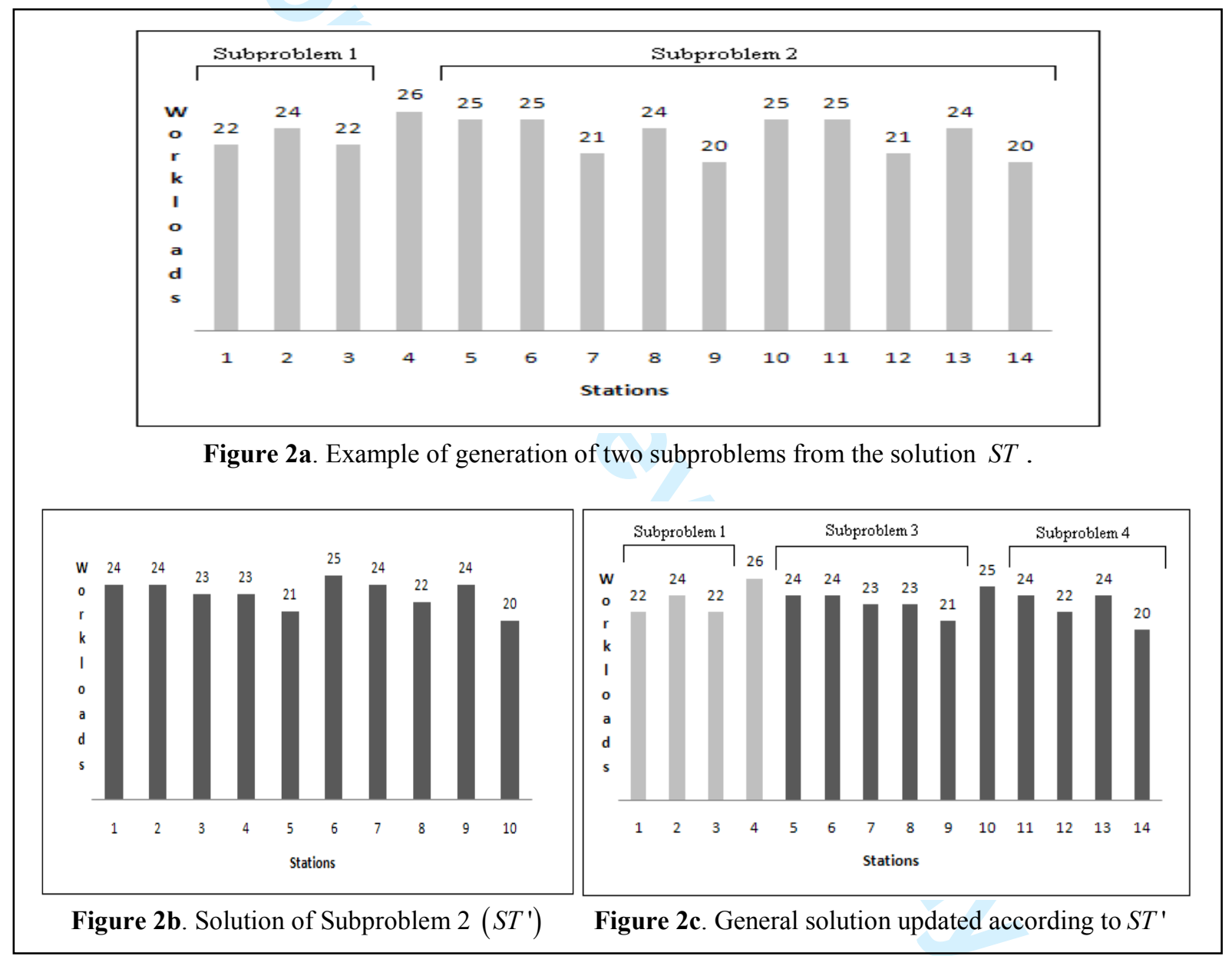

Figure 2. Example of generation of subproblems 
$0 . \quad S T:=$ Solve_SALBP-2 $\left(P R^{0}\right)$

1. $S T:=$ Local_search $(S T)$ and $S T^{*}:=S T$

2. $P R^{\prime}:=P R^{0}$ and $S T:=S T$

3. Set $P R:=\{\}$

4. id $\bar{f}_{s t}:=1$

5. $k:=\underset{j=1 . . P R^{\prime}, m}{\arg \max } W L[j]$, where $W L[j]:=\sum_{i \in P R^{\prime}, N: j=S T^{\top}[i]} P R^{\prime} . t_{i}$. If there is a tie, use the following criterion (the most central station): $k:=\underset{j=1 . . P R^{\prime} \cdot m: W[[j]=W[k]}{\arg \min }\left|j-\left(\frac{P R^{\prime} \cdot m+1}{2}\right)\right|$. If there is still a tie, then use lexicographical order.

6. If $(k \geq 2)$ then:

$P R \_l e f t:=$ Get_subproblem_left $\left(P R^{\prime}, S T^{\prime}, k\right)$

$c t$ _eft $:=\max _{j=1 . .1-1} W L[j]$

Add to Set_PR the triple element (PR_left, ct_left, id_fst)

If $\left(k \leq P R^{\prime} . m-1\right)$ then:

$P R \_$right : $=$Get_subproblem_right $\left(P R^{\prime}, S T^{\prime}, k\right)$

$c t_{-}$right $:=\max _{j=k+1 . P R^{\prime} \cdot m} W L[j]$

Add to Set_PR the tripe element ( $P R_{-}$right, ct_right, $\left.k+i d_{-} f_{s t}\right)$

7. If $\left(\operatorname{Set}_{-} P R=\{\overline{\}})\right.$ then return $S T^{*}$ and $\mathrm{END}$

8. $\left(P R^{\prime}, \bar{c}_{,}, i d_{-} f_{s t}\right):=\underset{\left(q, c^{\prime}, i d\right) \in \text { Set }_{-} P R}{\arg \max } c^{\prime}$. If there is a tie, the oldest generated subproblem is chosen. The element $\left(P R^{\prime}, c_{,}, i_{-} f_{s t}\right)$ is removed from Set_PR

9. $\quad S T^{\prime}:=$ Solve_SALBP-2 $\left(P R^{\prime}\right)$

10. $S T[i]:=S T^{\prime}[i]+i d_{-} f_{s t}-1 \quad \forall i \in P R^{\prime} . N$

11. If Local_search(ST) is best than $S T^{*}$ then go to Step 1. Else go to Step 5

Figure 3. Algorithm of the proposed heuristic

1. $\quad P R \_l e f t . N:=\bigcup_{j=1 . . k-1}\left\{i \in P R^{\prime} . N: j=S T^{\prime}[i]\right\}$

2. $\quad P R \_$left.t. $t_{i}:=P R^{\prime} . t_{i} \quad \forall i \in P R \_$left. $N$

3. $P R \_l e f t . P:=\left\{\left(i, i^{\prime}\right) \in P R^{\prime} . P: i, i^{\prime} \in P R \_\right.$left. $\left.N\right\}$

4. PR_left.m $:=k-1$

5. return $P R \_l e f t$

Figure 4. Algorithm of the function Get_subproblem_left $\left(P R^{\prime}, S T^{\prime}, k\right)$

1. $\quad P R \_$right. $N:=\bigcup_{j=k+1 . . P R^{\prime} \cdot m}\left\{i \in P R^{\prime} . N: j=S T^{\prime}[i]\right\}$

2. $\quad P R_{\text {_right. }} t_{i}:=P R^{\prime} . t_{i} \quad \forall i \in P R_{-}$right.N

3. $P R_{\text {_right }} P:=\left\{\left(i, i^{\prime}\right) \in P R^{\prime} . P: i, i^{\prime} \in P R_{-}\right.$right. $\left.N\right\}$

4. $\quad P R \_$right.m $:=P R^{\prime} . m-k$

5. return $P R_{-}$right

Figure 5. Algorithm of the function Get_subproblem_right $\left(P R^{\prime}, S T^{\prime}, k\right)$ 
0 . Let $S T$ the current solution (with $m$ ' workstations).

1. Let $k:=1$ and $p:=m$ '

2. Let $O S T$ the vector with the stations of the current solution $S T$ in which the stations are ordered decreasingly by their workload. In the case of tie, lexicographical order is used.

3. Let $n s t^{*}$ be the solution generated after applying $N E E$ with the rule N1 between the stations $O S T[k]$ and $O S T[p]$ to the current solution $S T$. If $n s t^{*}$ is better than $S T$, then $S T:=$ $n s t^{*}$ and go to Step 1 .

4. If $k=1$ and $p=2$, then return $S T$ and END.

5. If $k=p-1$ then go to Step 6; otherwise $k:=k+1$ and go to Step 3 .

6. $k:=1, p:=p-1$ and go to Step 2 .

\section{Figure 6. Local search procedure $L S P 1$}

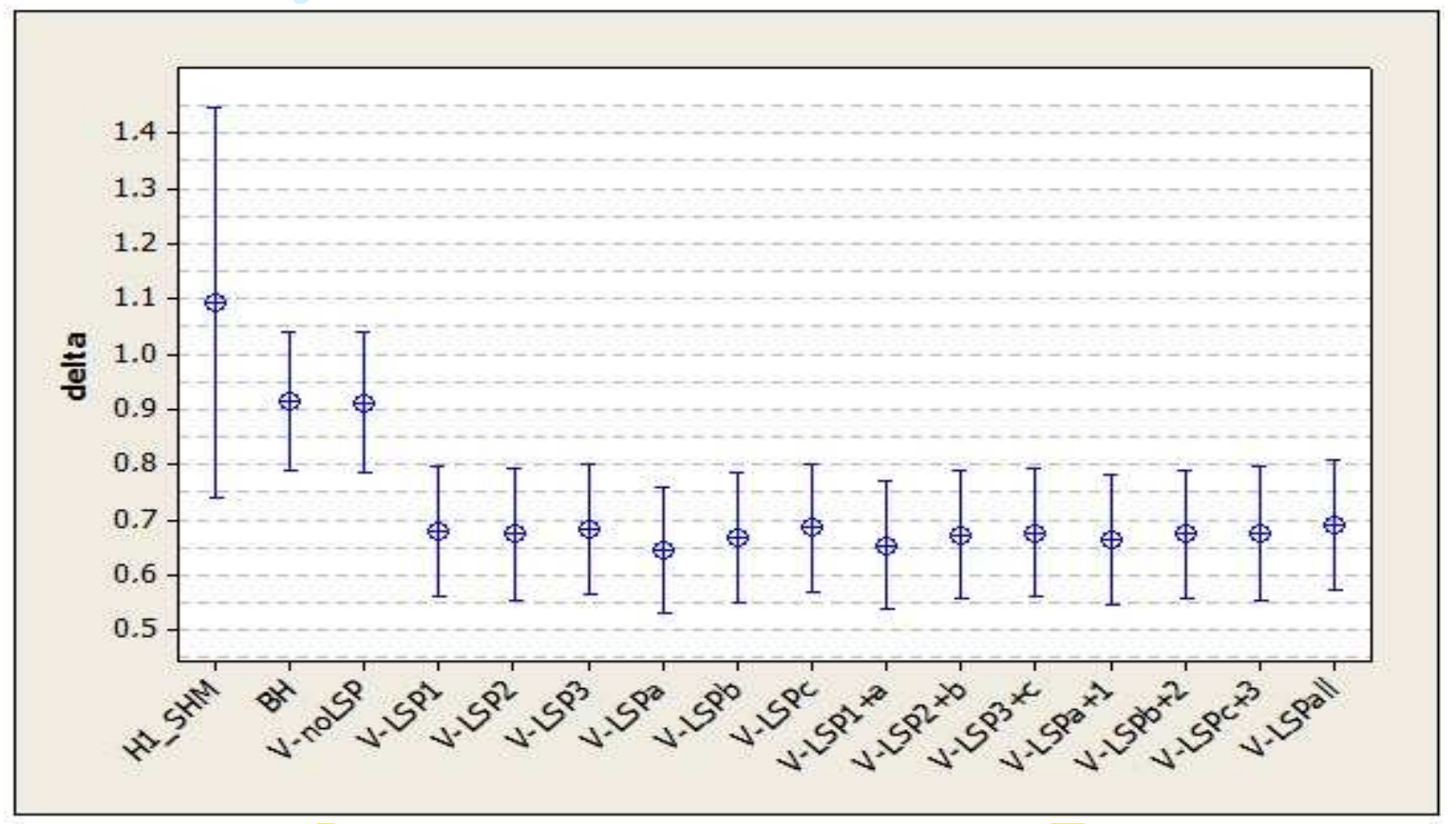

Figure 7. Delta means and 95\% LSD Intervals Graphic for the 16 algorithms

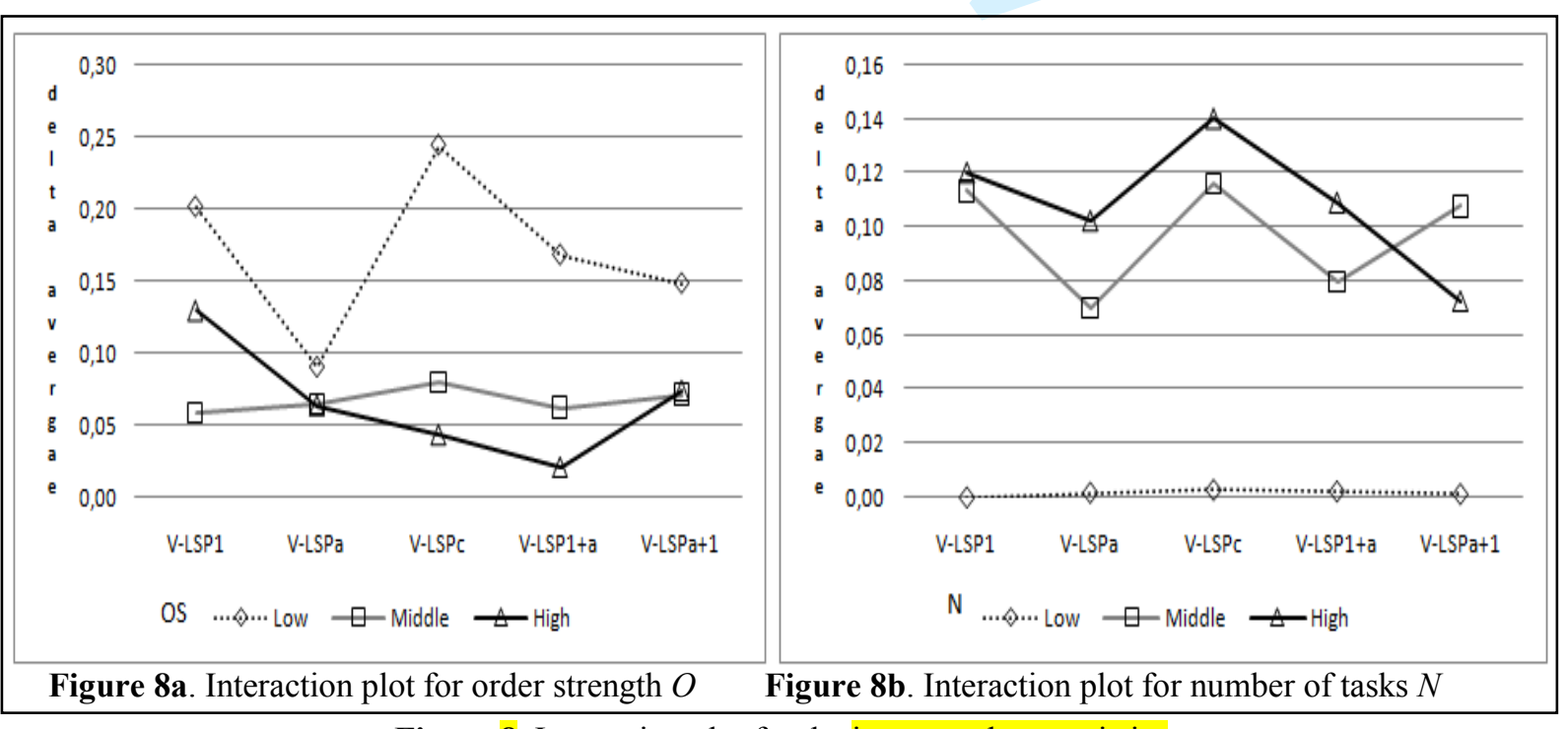

Figure 8. Interaction plot for the instance characteristics 